\title{
Projekt «Interne Qualitätskontrolle» des Aargauischen Ärzteverbandes
}

W. Schoop ${ }^{a}$, V. Heller ${ }^{b}$,

S. Morgenthaler ${ }^{b}$, F. Podzorski ${ }^{a}$,

M. Schär ${ }^{b}$

a Vorstandsmitglied des Aargauischen Ärzteverbandes

b Instruktorinnen Einführungskurs (MPA-Ausbildung des Kantons Aargau)
Die Finanzierung erfolgte ausschliesslich durch den Aargauischen Ärzteverband ohne irgendwelche zusätzliche Zuwendungen.

Korrespondenz:

Dr. med. Werner Schoop

Facharzt Allgemeinmedizin FMH

Leiter Arbeitsgruppe IQK

CH-5610 Wohlen

Tel. 0566223232

Werner.Schoop@hin.ch

\section{Zusammenfassung}

Der Artikel beschreibt ein Projekt, welches im Frühjahr bis Herbst 2004 durch den Aargauischen Ärzteverband in engster Zusammenarbeit mit den Instruktorinnen des Einführungskurses für Lehrlinge (Medizinische Praxisassistentin) durchgeführt wurde.

Bereits in den Jahren 2001/2002 wurde ein entsprechendes Pilotprojekt erarbeitet. Die Zielsetzung für 2004 bestand darin, konkrete Erkenntnisse im Umgang mit der internen Qualitätskontrolle (IQK) zu erhalten und zu dokumentieren und im Hinblick auf das geplante Obligatorium von internen Laborqualitätskontrollen zuhanden der QUALAB (Schweizerische Kommission für Qualitätssicherung im Labor) entsprechende Empfehlungen abzugeben.

Die Zielsetzung, der zeitliche Ablauf und die Ergebnisse werden dargestellt, die aufgetretenen Probleme analysiert und kommentiert, und es werden schlussendlich Empfehlungen formuliert, welche der QUALAB im Frühjahr 2005 zur Kenntnis gebracht wurden.

\section{Zielsetzung und Ablauf}

Im Anschluss an ein bereits 2001/2002 durchgeführtes Pilotprojekt war es das Ziel, konkrete Erfahrungen im Bereich der Planung, Durchführung und Dokumentation der internen Qualitätskontrolle primär im Labor, sekundär aber auch im Röntgen zu sammeln und die Erkenntnisse bezüglich interner Laborqualitätskontrollen der QUALAB zur Entscheidfindung für das geplante Obligatorium zur Verfügung zu stellen.

Die Erarbeitung eines im Pilotprojekt noch diskutierten eigenen aargauischen IQK-Handbuches wurde zurückgestellt, um Doppelspurigkeiten mit der QUALAB zu vermeiden.

Die Durchführung des Projektes erfolgte im Jahr 2004. Es wurden alle Praxen im Kanton Aargau, welche zu diesem Zeitpunkt einen Lehrling im 2. Lehrjahr hatten, zur Mitarbeit eingeladen.

Die Projekterarbeitung erfolgte in enger Zusammenarbeit mit den Instruktorinnen des Einführungskurses, wobei aber klar gewünscht wurde, dass das Projekt von den Ärztinnen und den diplomierten Praxisassistentinnen mitgetragen werden sollte.

\section{Projet «Contrôle de qualité interne» de l'Association des médecins argoviens}

Le présent article décrit un projet réalisé du printemps à l'automne 2004 par l'Association des médecins argoviens, en étroite collaboration avec les instructrices du cours d'introduction pour assistantes médicales.

Un projet-pilote de même type avait déjà été élaboré en 2001/2002. L'objectif pour 2004 consistait à approfondir la thématique du contrôle interne de qualité (CIQ), à en tirer des enseignements concrets et à les documenter. Tout cela aux fins d'émettre des recommandations à l'intention de la Commission suisse pour l'assurance qualité dans le laboratoire médical (QUALAB), chargée de coordonner les contrôles de qualité internes des analyses de laboratoire effectuées au cabinet du praticien.

Les auteurs présentent les objectifs, le déroulement et les résultats du projet, analysent les problèmes rencontrés et formulent des recommandations. Celles-ci ont été transmises à la QUALAB pour information au printemps 2005.

\section{Projektphasen und Ergebnisse}

\section{Rekrutierungsphase}

Im ganzen wurden 65 Praxen erfasst. Die Motivation zur Mitarbeit war anfänglich eher schwierig, was auch die Informationspolitik beeinflusste. Es musste zusätzliche Motivationsarbeit geleistet werden, was den Projektstart etwas verzögerte.

\section{Erfassung des Ist-Zustandes}

Gut 50\% der angeschriebenen Praxen haben mitgemacht. Es lässt sich aufgrund der Umfrageergebnisse annehmen, dass ein recht grosser Teil der Praxen auf freiwilliger Basis auch im 
Laborbereich eine interne Qualitätskontrolle eingeführt hat, dass aber eine nennenswerte Minderheit nur sehr sporadisch Kontrollen durchführt. Bei denjenigen Praxen, welche interne Laborkontrollen durchführen, sind vor allem die administrativen Abläufe (Kurzanleitungen für die Tests, Dokumentation usw.) teilweise ungenügend. Im Röntgen liegen die Probleme weniger bei den bereits obligatorischen Kontrollen als bei der Ausrüstung und deren korrekter Handhabung (Keile; Strahlenschutzmaterialien usw.)

\section{Erarbeitung der Arbeitsinstrumente}

Unter Anleitung der Instruktorinnen des Einführungskurses wurden im Laborbereich zwei verschiedene Arbeitsinstrumente ausgearbeitet. Einerseits wurden Kurzanleitungen für die jeweiligen Tests bzw. Geräte schriftlich niedergelegt, andererseits wurden Dokumentationsmöglichkeiten vorgeschlagen (im Bereich klinische Chemie schwergewichtig die von Dr. Fried vom Universitätsspital Zürich vorgeschlagene graphische Darstellung der IQK). Vor allem bei den Arbeitsanleitungen zeigte sich, dass die Praxisassistentinnen und die Lehrlinge wenig an die Ausarbeitung griffiger schriftlicher Arbeitsinstrumente gewöhnt sind. Hieraus resultierte ein vorübergehend erheblicher Aufwand für die Praxen. Es zeigte sich, dass auf allen Ebenen (vom Chef bis zum Lehrling) ein teilweise erhebliches Wissens- und auch Motivationsdefizit besteht, welches aber mit guter Information doch grösstenteils behoben werden konnte.

\section{Testphase}

Gut die Hälfte der ursprünglich zur Mitarbeit eingeladenen Praxen haben bis zum Schluss in der praktischen Durchführung mitgemacht. Aus Rücksicht auf den in der Anfangsphase doch hohen Aufwand haben wir uns entschlossen, lediglich einige wenige Laborwerte exemplarisch testen zu lassen. Die im Projekt geforderten Kontrollintervalle bewegten sich zwischen einem Tag (bei den Hämatologieautomaten) und zwei Wochen (bei der klinischen Chemie). Die abgelieferten Resultate inklusive deren Dokumentation zeigen eine breite Palette von ungenügend bis ausgezeichnet. Es bestätigen sich auch hier nochmals die Wissens- und Könnensgrenzen, besonders im Bereich der Dokumentation und Interpretation. Während der verhältnismässig kurzen konkreten Testphase vom Frühsommer bis Frühherbst 2004 konnte erwartungsgemäss nicht abgeschätzt werden, wie häufig tatsächlich Fehler durch die interne Kontrolle im Laborbe- reich aufgedeckt werden, welche nicht schon durch die externen Ringversuche zur Darstellung gelangten. Immerhin gibt es einzelne eindrückliche Beschreibungen von Abweichungen, die lediglich durch die interne Kontrolle, nicht aber durch die Ringversuche im entsprechenden Zeitabschnitt entdeckt wurden.

Im Röntgenbereich stellt sich die Durchführung der bereits seit längerem obligatorischen Kontrollen soweit problemlos dar; die korrekte und zuverlässige Anwendung der oben genannten Hilfsmittel (Keile, Strahlenschutzmaterialien) ist aber teilweise verbesserungswürdig.

\section{Auswertung des abschliessenden Fragebogens}

Insgesamt haben 41 Praxen (gut 60\%) den ausführlichen Fragebogen ausgefüllt und zurückgeschickt. Die Zielsetzung des Projekts wurde von einer nennenswerten Minderheit als unklar empfunden, wobei hier der Verdacht besteht, dass teilweise Motivationsprobleme zugrunde lagen. Erfreulicherweise werden die im Projekt angeregten Kurzarbeitsanleitungen und Dokumentationsmöglichkeiten schliesslich als gut bis sehr gut bewertet. Eine überwiegende Mehrheit der Praxen gibt an, dass die IQK gemeinsam durch die Lehrlinge und die diplomierten Praxisassistentinnen durchgeführt werden. Hier bestehen aber gewisse Diskrepanzen zu den direkten Aussagen von Lehrlingen. Möglicherweise ist die Absicht besser gewesen als die tatsächliche Durchführung; viele Lehrlinge fühlten sich alleine gelassen durch die Lehrmeister/innen. Erfreulich ist die Tatsache, dass eine Mehrheit der Praxen auch Wartungs- und Kontrollisten führt. Gewisse Zweifel bestehen aber auch hier bezüglich der konsequenten Anwendung und Durchsetzung. Dasselbe gilt für allfällige Listen möglicher Fehlerquellen, welche allerdings nur in relativ wenigen Praxen existieren. Die Ansichten über die im Projekt geforderten Kontrollintervalle bei der Labor-IQK sind äusserst unterschiedlich. Eine solide Mehrheit ist aber mit den Kontrollintervallen von einem Tag für die automatisierte Hämatologie und zwei Wochen für die klinische Chemie einverstanden. Der durch die Teilnehmer geschätzte Zeitaufwand beträgt für die interne Laborqualitätskontrolle pro normalen Arbeitstag etwa 20 Minuten. Der geschätzte finanzielle Aufwand ist schwierig zu erheben, da diverse Praxen hier keine Antwort gegeben haben. Der Betrag scheint sich aber doch im Bereich von mehreren Hundert Franken pro Monat zu bewegen. Eine Mehrheit der Praxen befürwortet grundsätzlich die Weiterführung der internen 
Laborqualitätskontrolle; die Ansichten zur vernünftigen Kontrollfrequenz bleiben aber sehr breit gestreut.

\section{Erkenntnisse}

1. Trotz anfänglicher Motivationsprobleme vieler Teilnehmer kann im Vergleich zum Pilotprojekt 2001/2002 eine erhebliche Verbesserung der Motivation und auch eine deutliche Steigerung der Qualität der abgegebenen Resultate festgestellt werden. Das Bewusstsein um die Wichtigkeit der internen Qualitätskontrollen (sowohl im Röntgen wie auch im Labor) scheint unabhängig von einem allfälligen Obligatorium deutlich im Steigen zu sein.

2. Unterstützung bzw. bessere Ausbildung ist besonders im Bereich der konkreten Arbeitsanleitungen für Labortests und Geräte und bei der Dokumentation der internen Qualitätskontrolle angezeigt. In der jetzigen Ausbildung der medizinischen Praxisassistentin kommt das Thema IQK eher zu kurz.

3. Sowohl der zeitliche als auch der finanzielle Aufwand für die Laborkontrollen bewegen sich zwar in einem grundsätzlich vernünftigen, aber insgesamt doch keinesfalls zu vernachlässigenden Bereich. Unserer Meinung nach sollte sich der Zeitaufwand aber mit zunehmender Erfahrung und guten Arbeitsinstrumenten noch reduzieren lassen. Diese Kennzahlen sind selbst durch unsere umfangreiche Befragung noch nicht mit genügender Sicherheit festzulegen und bedürfen weiterer genauer Berechnungen (insbesondere, was die finanzielle Seite angeht!).

\section{Ergänzende Bemerkungen}

Es gilt zu berücksichtigen, dass die interne und externe Qualitätskontrolle alleine die Probleme im Praxislabor noch nicht löst. Häufig ist es ausgesprochen mühsam und frustrierend, Fehlerquellen auf der technischen Ebene zu suchen. Die Liefer- und Wartungsfirmen sind häufig ebenfalls überfordert.

Besonderes Augenmerk ist auch der Tatsache zu widmen, dass die Streubreiten gewisser Laboruntersuchungen (auch bei den entsprechenden internen Kontrollen) enorm gross sind, was möglicherweise vielen Beteiligten nicht bewusst ist (ein Beispiel: INR-Werte mit einer Streubreite von 2,1 bis 3,9 [= 2 Standarddeviationen]). Das Bewusstsein um diese Tatsache dürfte den Umgang mit Laborresultaten nachhaltiger beeinflussen als manche Kontrollen. Leider sind die relativen Häufigkeiten der bei Qualitätskontrollen festgestellten Fehler nicht bekannt (Präanalytik?
Geräteseitige Probleme? Probleme mit Testreagenzien? usw. ). Grundsätzlich hätte ein solches Wissen Konsequenzen für die Ausgestaltung der (Labor-)IQK sowohl hinsichtlich Häufigkeit/ Kontrollfrequenz als auch bezüglich des jeweiligen Durchführungszeitpunkts.

\section{Empfehlungen der Arbeitsgruppe IQK des Aargauischen Ärzteverbandes}

Diese Empfehlungen wurden vom Vorstand des Aargauischen Ärzteverbandes aufgrund des vorliegenden Schlussberichtes der Arbeitsgruppe IQK am 7. April 2005 verabschiedet und insbesondere der QUALAB zugänglich gemacht; die nun vorliegende, redaktionell bearbeitete Version wird ebenfalls offiziell an die QUALAB weitergeleitet.

\section{Allgemeines}

1. Bevor ein Obligatorium für interne Qualitätskontrollen beschlossen wird, muss das genaue Konzept zur konkreten Umsetzung bekannt sein (besonders auch bezüglich des allfälligen Ausbildungsbedarfs und der entsprechenden Angebote sowie Kontrollmechanismen/Sanktionskriterien).

2. Der Motivierung und Information ist erste Priorität einzuräumen. Vor allem muss im Laborbereich bewusstgemacht werden, dass Fehler nicht unbedingt derart offensichtlich erkannt werden können, wie dies beispielsweise bei einem Röntgenbild meist möglich ist.

Die Information und Motivation sollen via die kantonalen Ärztegesellschaften und die Fachgesellschaften - also auf dem ärztlichen und nicht auf dem administrativen Weg - erfolgen.

3. Die IQK soll in erster Priorität als Selbstlernsystem und nicht primär als Selektionsinstrument geplant werden.

\section{Labor}

1. Bevor die interne Qualitätskontrolle obligatorisch eingeführt wird, müssen die Ausbildungsreglemente der Praxisassistentinnen (Ziel, Inhalt, Zeitrahmen der Ausbildung in IQK) überprüft und gegebenenfalls angepasst werden.

2. In der Ausbildung ist ein Schwergewicht auf das Erarbeiten von griffigen Kurzanleitungen zu Geräten und Tests sowie auf die Dokumentation zu legen.

3. Es darf nicht vergessen werden, dass bei der Einführung eines IQK-Obligatoriums Praxen ohne Lehrlingsausbildungsfunktion eventuell einen zusätzlichen Support brauchen. 
4. Die kommenden «offiziellen» IQK-Bestimmungen müssen mit industrie-/lieferantenseitigen Vorschriften abgeglichen werden; es darf hier keine Widersprüche geben.

\section{Röntgen}

1. Die Fortsetzung der bisherigen obligatorischen Kontrollen scheint sinnvoll und machbar.

2. Ein besonderes Augenmerk ist auf die kleineren Ausrüstungsgegenstände und deren $\mathrm{Zu}$ stand (Gonadenschutz, Keile usw.) zu legen.

3. Bei der Aufnahmetechnik ist ein Schwerpunkt auf die konsequente Anwendung allgemein anerkannter Einstellungskriterien zu legen.
Weitere Aktivitäten der Arbeitsgruppe IQK des Aargauischen Ärzteverbandes

Die Arbeitsgruppe IQK bemüht sich, sobald als möglich die Homepage des Aargauischen Ärzteverbandes für die Kommunikation von IQKBelangen zu nützen. In erster Priorität sollen Beispiele für Arbeitsanleitungen und Dokumentation, dann aber auch Listen möglicher Fehlerquellen usw. zur Verfügung gestellt werden.

Die Arbeitsgruppe IQK des Aargauischen Ärzteverbandes bleibt vorderhand bestehen und ist im Aargauischen Kantonalverband Ansprechpartner besonders auch im Zusammenhang mit einer späteren obligatorischen Einführung der internen Qualitätskontrolle im Labor. 